\title{
MANETS and Internet of Things: The Development of a Data Routing Algorithm
}

\author{
I. A. Alameri \\ Computer Center \\ Jabir Ibn Hayyan Medical University \\ Iraq \\ Ib.alameri@jmu.edu.iq
}

\begin{abstract}
Internet of things (IoT), is an innovative technology which allows the connection of physical things with the digital world through the use of heterogeneous networks and communication technologies. IoT in smart environments interacts with wireless sensor network (WSN) and mobile ad - hoc network (MANET), becoming even more attractive and economically successful. Interaction between wireless sensor and mobile ad - hoc networks with the internet of things allows the creation of a new MANET - IoT systems and IT - based networks. Such systems give the user greater mobility and reduce costs. At the same time new challenging issues are opened in networking aspects. In this work, author proposed a routing solution for the IoT system using a combination of MANET protocols and WSN routing principles. The presented results of solution's investigation provide an effective approach to efficient energy consumption in the global MANET - IoT system. That is a step forward to a reliable provision of services over global future internet infrastructure.
\end{abstract}

Keywords-IoT; MANET; dynamic routing; wireless sensor network

\section{INTRODUCTION}

Internet of Things (IoT) and cloud computing [1] represent a part of the future internet paradigm. The capability of objects (like physical or virtual things) to identify and communicate with each other at any time with evolving communication technologies gives the possibility to provide advanced services over global infrastructure in different areas of everyday life [2]. The interconnection of smart objects and its interoperability with global communications serve as a main incorporated idea in Internet of Things (IoT) systems. In an IoT system, a major role is played by the wireless sensor network (WSN) as its components comprise: sensing, data acquiring, heterogeneous connectivity and data processing. A wireless, self-configuring and multi-hop network is called mobile ad-hoc network (MANET) [3]. Each MANET node operates as a router or as a network end system and is closely related to WSNs. The interaction between MANET and IoT opens new ways for service provision in smart environments and challenging issues in its networking aspects. One of the important factors in MANET-IoT systems is the energy balancing over nodes, since the IoT system is based mostly on many different wireless sensors and selection from MANET protocols focuses on the most efficient and shortest routes. A proper utilization of sensor's battery power is a significant key in maintaining network connectivity of a multihop wireless network. Because of this, many researchers are focusing on designing energy efficient routing protocols that prolong network's lifetime. Wireless network protocols cannot be used directly due to resource constraints of sensors' nodes, computational speed, and human interface with node's devices and network node density. Therefore, there is a need of composite solution for routing over MANET-IoT networks, which can use node residual energy efficiently and extend the network lifetime.

In this paper, an algorithm of energy efficient and safe-weighted clustering routing for the mobile IoT system using a combination of MANET and WSN routing principles is proposed. Clustering is a method of making routing less complex, and for some sensor networks, more energy efficient. Such combination of MANET and WSN routing principles is able to increase the sensors lifetime in the overall mobile IoT system. There is importance in deciding on how many cluster heads (CHs) are needed and which sensor node shall act as the cluster head. MANET network nodes were chosen as a cluster head and a proactive routing protocol was used in such a way that it is possible to control and update a table of information about the network state. Nodes that lose their energy rapidly and are ended up with low energy were recognized and their workloads were limited for transactions. All investigations for the selection of a routing path over the MANET-IoT system were performed using Matlab. This research work provides important key insights into the combination of MANET and WSN routing principles by increasing the sensors lifetime in the overall IoT system.

\section{LITERATURE REVIEW}

\section{A. Manet}

MANETs are independently self-organized networks without fixed topology. In such a network, each node acts both as a router and host at the same time. All network nodes are equivalent to each other and can join or move out freely in the network. Mobile nodes present in the radio range of each other can communicate directly and transfer the necessary 
information. All network nodes have a wireless interface to interact with each other. This kind of network can work anywhere and it is fully distributed without the assistance of any infrastructure as base stations or access points. There are two multiple ad-hoc network types: i) MANET and ii) mobile ad-hoc sensor network. A mobile ad-hoc sensor network has much wider sequence of operations and requires less complicated setup steps in comparison with typical sensor networks which directly communicate with a centralized controller [5]. There are six main characteristics of MANETs [4]: dynamic topology, distributed operation, lightweight terminals, multi-hop routing, and shared physical medium and autonomous terminal. Routing MANET protocols can be categorized into three general categories:

\section{1) Topology-based routing}

The routing types [5] are: (a) proactive routing protocols (routing table-based), (b) reactive routing protocols (demand based) (c) hybrid routing protocols which consist of combinations of reactive and proactive protocols. One of them is ZRP (zone routing protocol).

\section{2) Location-based routing}

To make routing decision, the actual position of nodes is used by the location-based routing in any area. For example, by using global positioning system (GPS), the information of location can be obtained. Location-aided routing (LAR) protocol is an example of location-based routing.

\section{3) Energy awareness based routing}

Each network node supports multiple entries in routing tables. For choosing the optimal route, assessing power levels of network nodes is available. In this case, routing table corresponds to the nodes power level and is maintained by transferring relevant messages. The number of reachable nodes is determined by the number of entries in the routing table of nodes by using the power level. Thus, the number of entries in routing tables gives the total number of network nodes [6].

\section{B. Internet of Things (IoT)}

The need to "recruit" all things that surrounds us was a great reason for the connection of electronics, devices, with digital communications, using internet as the main data transmission medium [7]. All communication, management and information exchange are processing among connected things and objects. The capability of real or virtual things and objects to be identifiable, to communicate and to interact allows us to build networks of interconnected objects, end users or other entities in the global internet network [9]. So the term "Internet of Things" mainly means the global infrastructure of interconnected things, devices, or objects, which can communicate, actuate, exchange their information over internet to the end users by using the interaction between communication technologies and networks.

\section{The Interaction between Internet of Thing and MANET}

Possibilities of wide application of IoT systems in different areas are directly dependent on the opportunities of interoperability between different communication technologies and networks in smart environments [8]. The growth of sensors quantity leads to the increasing human need for remote monitoring of different processes. And this became possible by the widespread deployment of wireless sensor networks (WSN). Basically, WSN is a network, which consists of different sensors that are autonomously capable to read information from the measured object, to handle sensed data, temporarily store it and transfer them to another network node, which is also a sensor. As WSN is a normally centralized network [12], so the data, sensed and transferred from other sensors, are transmitted to the central node, which is usually called sink. In this manner, the wireless sensors are able to communicate with each other and thus open very wide usability opportunities of wireless sensor networks in IoT systems. Wireless sensor networks mainly are the basic element in the global IoT system, as sensors have the ability to gather information from different things and transmit it over the network. However, the reliability of IoT systems is highly dependent on the power consumption and scalability of WSN [13]. Sensors should transmit measured data so efficiently to the sink, that their battery energy would be used at minimum level. Because of this, WSN should easily accommodate network changes. This is related to the lifetime of WSN as well, as low or empty battery leads to the death of sensors. In this way, the routing principles and methods are very important and challenging issues of WSN as data should be transmitted by another sensor and the dead sensor should be eliminated from the routing path. These should be done with respect to Quality of Service (QoS) over WSN [14].

Wireless sensor network in general is similar to MANET since both are multi-hoped and self-organized networks. However, the topology of MANET is more changeable than WSN. MANET protocols can allow it to act as a WSN backbone [15] and access wireless sensor network's nodes as well as exchange information with WSN about MANET entry points [10]. These two networks can enable more effective and reliable cross-network routing in the IoT context. The intersection of MANET, WSN and IoT will be addressed as MANET-IoT system, which is discussed in detail in Section III.

Networking in the MANET-IoT system is based on MANET routing protocols, WSN routing principles and data sensing, handling and processing using IoT. In general, the networking of such a system is very challenging regarding routing aspects. Also, it is related to system mobility and limited network sensor resources. Most of MANET protocols are designed with focus on QoS $[16,17]$ and routing in WSNs is focused on the efficient node energy consumption [18]. The connection of different things with limited features to the internet and the interaction with different wireless and mobile ad-hoc networks must guarantee connectivity, accessibility and reliability of the MANET-IoT system in smart environments. The solutions for the routing protocols of ad-hoc network modification in order to fulfil the requirements of the IoT were presented in [19]. Routing principles were changed by integrating IPv6 [20]. However, the interaction of IoT with MANET and WSN requires new, optimized solutions for data routing in the MANET-IoT system. Author proposes an algorithm for data routing, which is mainly focused on energy 
efficiency and safe weighted clustering in the MANET-IoT system. The solution is described in Sections III and IV.

\section{PROPOSED MANET-IOT SYSTEM DATA ROUTING ALGORITHM}

In order to describe complex networks like WSN, graphs are a suitable model. It can be assumed that WSN, in creating energy aware route selection schemes is a graph with edges representing communication links between vertices and vertices representing sensor nodes. A node's residual energy is denoted by the weight on a vertex while the amount of energy that a node needs to transfer a unit of information along the edge is indicated by the weight on an edge [31]. The lowest energy level of any node on the route is called the residual energy. The sum of the weights on all the edges present on the route is the energy consumed along the route. The best and suitable energy aware route selection scheme for WSN is to use the nodes with higher energy levels in such a way that the total energy consumed along the data forwarding path is reduced.

The proposed algorithm adopts dynamical monitoring, which controls the energy of the cluster heads, and a predefined threshold value. The purpose of this monitoring mechanism is the transferring of cluster head based on the comparison result between the threshold value and the cluster head's energy. The algorithm presented in Figure 1 has three phases: setup, steady and threshold. First step is a cluster head selection. All the chosen cluster heads send a message of advertisement to all the non-cluster head nodes after the selection of the cluster head. The non-cluster head nodes choose their cluster heads for the current round based on the received signal strength of the message advertised and send back a request message to the selected cluster heads informing their membership that leads to the formation of cluster. The message sent to the cluster heads includes the node's ID and the location of the sender node. When all nodes are deployed, the network starts to select the cluster heads and carry out clustering and layering. Then, the nodes begin to periodically start data collection and transmit them to the sink node. With the change of time, the network topology structure is also changing. If cluster head energy is lower than the predefined threshold value, the third loop is applied to replace cluster head by another node, which poses the largest energy within the cluster.

The new cluster head continues to cooperate with cluster members. This way protects cluster heads with lower energy. This mechanism can protect cluster heads from quick death and prolong the network lifetime. When we have all the information about network and nodes, then we are choosing the routing method for transmission information. For the evaluation of network lifetime three route path selection methods are used: NP (node place), BST (node battery state) and ER (energy resource). The NP aims to find the route with minimum hop count and for searching nodes, node location parameters or methods are used (RSSI, AoA and ToA). The cluster head evaluates all neighbor nodes in the cluster. If the information does not satisfy required criteria, cluster heads send message to the neighbor cluster head to help find a route to the sink. BST selects node with higher battery state. Using the ER method we calculate all network energy resources using the proposed algorithm. In the new algorithm, a threshold value is added in order to monitor the node's energy.

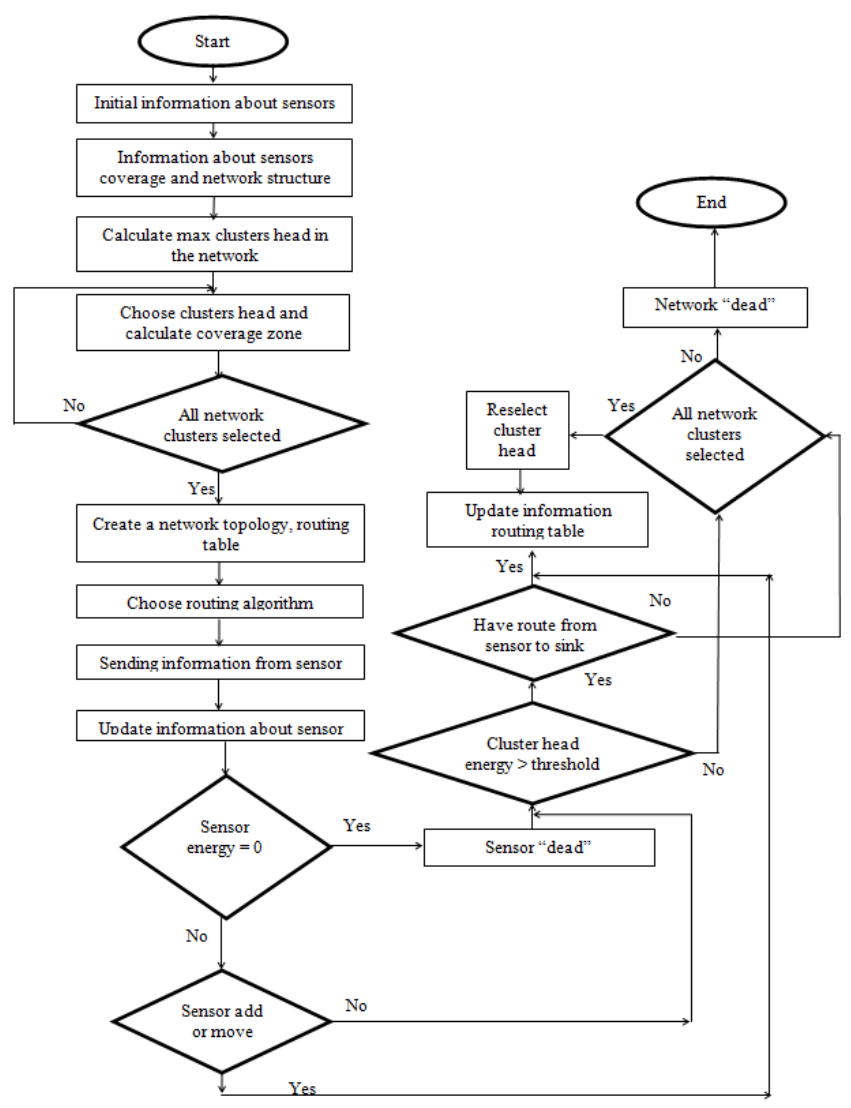

Fig. 1. Proposed common route choosing algorithm

\section{A. Performance evaluation of routing algorithm}

Simulation experiments are carried out using Matlab. The principal goal of these simulations is to analyze our algorithm and compare it with others. For analyzing and comparing the performance of our proposed method we used two metrics: node energy and network lifetime (or number of rounds). Network lifetime is one of the main characteristics to evaluate sensor network performance. Such a parameter includes coverage, connectivity and node availability. Network lifetime $T_{n}$ is defined as the time when sensor network loses connectivity. The route lifetime is defined by the first node failure:

$$
T_{\text {route }}^{n}=\min T_{\gamma}, \gamma \varepsilon N
$$

where $T_{\gamma}$ is the lifetime of node $\gamma$ in ij-route.

We tested the proposed algorithm using an initial number of alive sensors $N=17$, each with a range $d=8 \mathrm{~m}$. We used a network of size $M=20 \times 20 \mathrm{~m}$, with a sink located at point coordinates $[x=7, y=18]$. According our proposed solution, the optimal number of cluster was first calculated. As shown in Figure 2, in this case the optimal numbers of clusters are 3 . The network at which we apply our tests is shown in Figure 3. The 
green round circle denotes the sink. The blue round circle denotes the cluster heads. The red and yellow round circles denote the sensors, but red can be the cluster head also. The connection line denotes the path of a single hop from the sensor nodes to the cluster head. In the first scenario, sensors are sending information to the sink over three cluster heads. Figure 10 presents the simulation results (a) network lifetime, (b) each node battery energy and (c) dependency of node energy on the number of rounds.

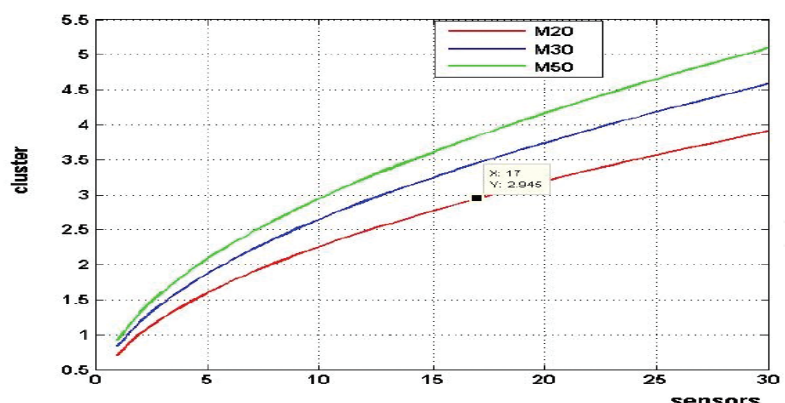

Fig. 2. Optimum number of clusters versus sensors and network size.

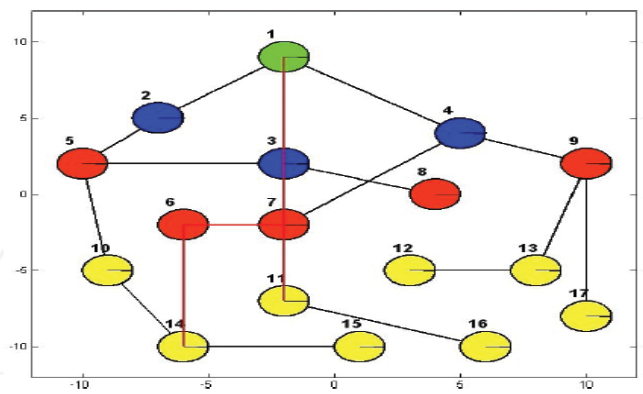

Fig. 3. The test network structure.

As can been seen in Figure 4, using such information to the routing method network lifetime (dimension is days) is 216 , and the fastest losing power nodes 3 and 7. During the analysis of this data, we observe that the consumption of energy distribution is unbalanced in the network and observable the weakness network location. The next simulation step was to use routing change over the simulation period, when the node energy level is lower than the threshold value. Figure 5 shows that after 90 rounds (a), the node 4 energy level is lower than the threshold value, therefore the sending information form node 7 we redirect from node 4 to node 3 and from nodes 11-7 to nodes 11-8. The next time (b) after 192 round we change other routes. Using this algorithm, our simulation network lifetime was 368 (c). The energy parameters are shown in Figure 6. For evaluation of the effectives of our proposed algorithm, further simulations were carried out. The simulation results are presented in Figures 7 and 8. By comparing the results, we found that by using our proposed algorithm, the network lifetime is the longest than using simple or clustering without weight routing methods. The main objective of the dynamic cluster head rotation mechanism is to evenly distribute the energy load among all the sensor nodes so that there are no overly utilized sensor nodes that will run out of energy before the others. And we can see that the distribution of network nodes' energy consumption becomes smoother (Figure 8). The assumptions made for compare different routing are as follows: network nodes are homogeneous and not mobility; they are equipped with power control; have active and sleep mode; each sensor has a unique identifier and uniformly deployed over the target area to continuously monitor the environment.

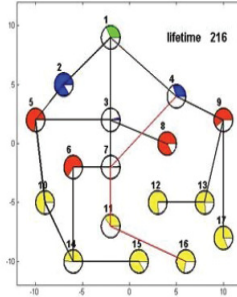

a)

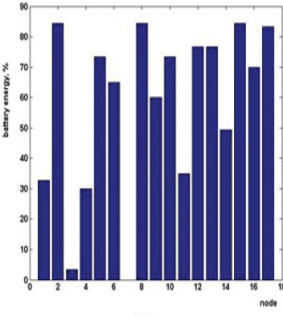

b)

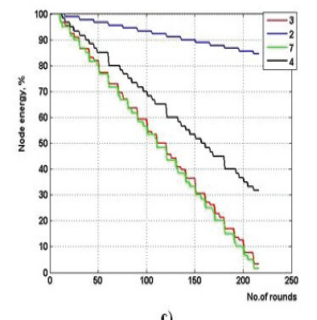

Fig. 4. Simulation results (a) network lifetime, (b) each node battery energy (c) dependency of node energy on the number of rounds.

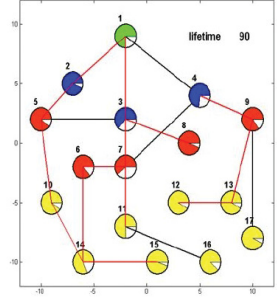

a)

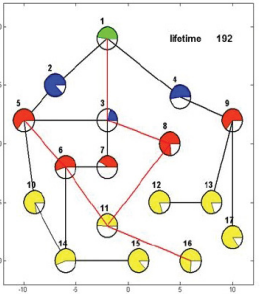

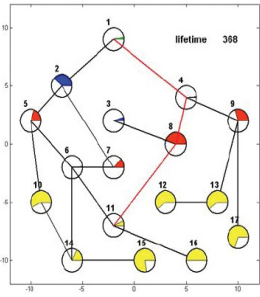

Fig. 5. WSN lifetime using a node energy level threshold value for routing change.
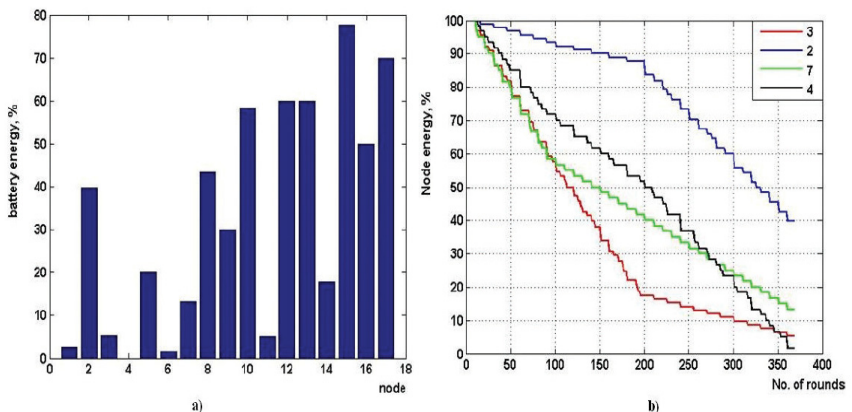

Fig. 6. WSN energy parameters

\section{CONCLUSION}

In this paper, a proposed algorithm of energy efficient and safe-weighted clustering routing for the mobile IoT system using a combination of MANET and WSN routing principles is presented. The clustering method is chosen because sensor nodes in a network organize themselves hierarchically. The simulation results show that if we use the combination method for information routing in the wireless sensor network, we increase sensor lifetime in the overall IoT system. Because we 
used dynamical cluster head selection, the weighting factors are added for routing from the sensor to the sink. When the network is heterogeneous and mobile, using routing weight is very important mainly due to the network's dynamic topology. Weight function was used to calculate each sensor's node value and thus the cost of all routes.

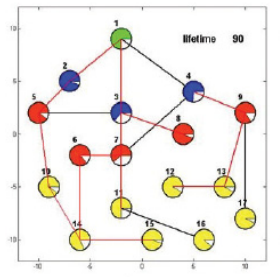

a)

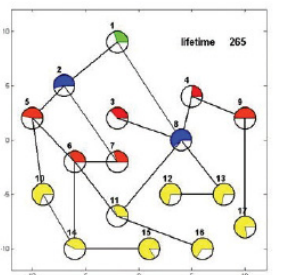

d)

Fig. 7. WSN lifetime using the proposed algorithm
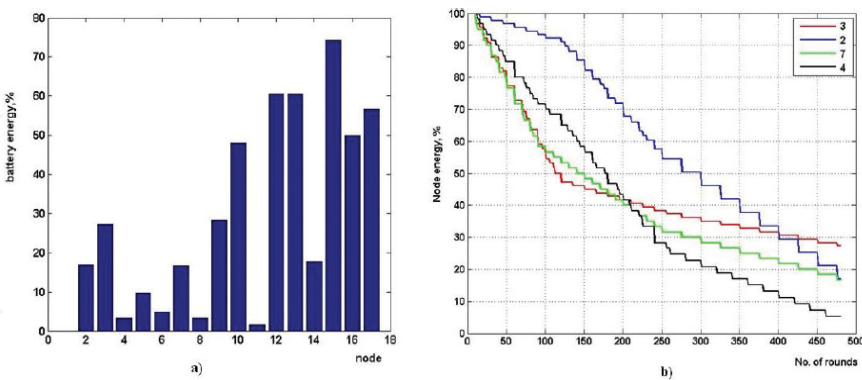

Fig. 8. WSN energy parameters using the proposed algorithm

\section{REFERENCES}

[1] I. A. Alameri, G. Radchenko, "Development of Student Information Management System based on Cloud Computing Platform" Journal of Applied Computer Science \& Mathematics, Vol. 11, No. 2, pp. :4-19, 2017

[2] ITU-T/Recommendation Y.2060, Overview of the Internet of Things, available at: https://www.itu.int/rec/T-REC-Y.2060-201206-I, 2013

[3] M. B. M. Kamel, I. Alameri, A. N. Onaizah, "STAODV: A Secure and Trust based Approach to Mitigate Blackhole Attack on AODV based MANET", IEEE 2nd Advanced Information Technology, Electronic and Automation Control Conference, pp. 1278-1282, 2017

[4] S. Kumar, S. Kumar, "Study of MANET: Characteristics, Challenges, Application and Security Attacks", International Journal of Advanced Research in Computer Science and Software Engineering, Vol. 3, No. 5, pp. 252-257, 2013

[5] Z. Hussain, R. Balakrishna, "A survey on Manets-Types, Characteristics, Applications and Protocols used", National Conference on Frontiers and Advances in Information Science and Technology, 2013.
[6] P. Nayak, R. Agrawal, S. Verma, "Energy Aware Routing Scheme for Mobile Ad Hoc Network Using Variable Range Transmission", International Journal of Ad Hoc, Sensor \& Ubiquitous Computing, Vol 3, No. 4, pp. 53-63, 2012

[7] S. Agrawal, M. Lal Das, "Internet of Things - A Paradigm Shift of Future Internet Applications", Nirma University International Conference on Engineering; pp. 1-7, 2011

[8] O. Vermesan, P. Friess, (eds), Internet of Things: Converging Technologies for Smart Environments and Integrated Ecosystems, River Publishers, 2013

[9] P. Suresh, J. Vijay Daniel, V. Parthasarathy, R. H. Aswathy, "A State of the Art Review on the Internet of Things (IoT) History, Technology and Fields of Deployment", International Conference on Science Engineering and Management Research, pp. 1-8, 2014

[10] D. Miorandi, S. Sicari, F. De Pellegrini, I. Chlamtac, "Internet of Things: Vision, Applications and Research Challenges", Ad Hoc Networks, Vol. 10, No. 7, pp. 1497-1516, 2012

[11] J. Gubbia, R. Buyyab, S. Marusica, M. Palaniswamia, "Internet of Things (IoT): A Vision, Architectural Elements, and Future Directions", Future Generation Computer Systems, Vol. 29, No. 7, pp. 1645-1660, 2013

[12] P. Bellavista, G. Cardone, A. Corradi, L. Foschini, "Convergence of MANET and WSN in IoT Urban Scenarios", IEEE Sensors Journal, Vol. 13, No. 10, pp. 3558-3567, 2013

[13] G. S. Yovanof, G. N. Hazapis, "An Architectural Framework and Enabling Wireless Technologies for Digital Cities \& Intelligent Urban Environments", Wireless Personal Communications, Vol. 49, No. $3 \mathrm{~m}$ pp. 445-463, 2009

[14] N. Bessis, F. Xhafa, D. Varvarigou, R. Hill, M. Li, (eds), Internet of Things and Intercooperative Computational Technologies for Collective Intelligence, Springer-Verlag, 2013

[15] M. Potnuru, P. Ganti, Wireless Sensor Networks: Issues, Challenges and Survey of Solutions, available at: https://www.academia.edu/890321 /Wireless_Sensor_Networks_Issues_Challenges_and_Survey_of_Solutio ns

[16] B. Bhuyan, H. Kumar, D. Sarma, N. Sarma, A. Kar, R. Mall, "Quality of Service (QoS) Provisions in Wireless Sensor Networks and Related Challenges", Wireless Sensor Network, Vol. 2, No. 11, pp. 861-868, 2010

[17] M. Rath, U. P. Rout, "Analysis and Study of Security Aspect and Application Related Issues at the junction of MANET and IoT", International Journal of Research in Engineering and Technology, Vol. 4, No. 13, pp. 426-430, 2015

[18] A. Boukerche, B. Turgut, N. Aydin, M. Z. Ahmad, L. Boloni, D. Turgut, "Routing Protocols in Ad Hoc Networks: A Survey", Computer Networks, Vol. 55, No. 13, pp. 3032-3080, 2011

[19] L. Hanzo, R. Tafazolli, "A Survey of QoS Routing Solutions for Mobile Ad hoc Networks", IEEE Communications Surveys \& Tutorials, Vol. 9, No. 2, pp. 50-70, 2007

[20] K. Akkaya, M. Younis, "A Survey on Routing Protocols for Wireless Sensor Networks", Ad Hoc Networks, Vol. 3, No. 3, pp. 325-346, 2005

[21] Y. Tian, R. Hou, "An Improved AOMDV Routing Protocol for Internet of Things", International Conference on Computational Intelligence and Software Engineering, pp. 10-12, 2010

[22] T. Tsvetkov, "RPL: IPv6 Routing Protocol for Low Power and Lossy Networks", Network Architectures and Services, Vol. 2011, No. July, pp. 59-66, 2011

[23] T. Chan, C. Chen, Y. Huang, J. Lin, T. Chen, "Optimal Cluster Number Selection in Ad-hoc Wireless Sensor Networks", WSEAS Transactions on Communications, Vol. 7, No. 8, pp. 837-846, 2008 